\title{
Formation of Oxide Nanostructures Investigated by In Situ UHV-TEM
}

\author{
L. Sun,* J. C. Yang* \\ * Department of Materials Science and Engineering, University of Pittsburgh, Pittsburgh, PA \\ 15261
}

In the rapidly developing field of nanotechnology, the controlled formation of oxide nanostructures is technologically important for their potentially novel optical, magnetic and sensor properties. Oxidation can be viewed as a processing tool for creating such self-ordered nanostructures. However, so far few studies exist concerning the initial oxidation stage-from the nucleation to the initial growth of metal oxides. Hence, we have used in situ ultra high vacuum transmission electron microscopy (UHV-TEM), to study the initial stages of oxidation, in order to gain insights into the oxidation kinetics with controlled surface conditions.

We have studied the oxidation of $\mathrm{Cu}(100)$ at temperatures below $350{ }^{\circ} \mathrm{C}$ in order to extend our previous studies, which centered on oxidation mechanisms that occurred at temperatures at and above $350^{\circ} \mathrm{C}$ [1]. For the temperature range we examined $\left(200\right.$ to $\left.350{ }^{\circ} \mathrm{C}\right)$, epitaxial $\mathrm{Cu}_{2} \mathrm{O}$ islands form with a triangular in cross-section shape that had rounded edges when $\mathrm{Cu}(100)$ was exposed to dry oxygen at $5 \times 10^{-4}$ torr in situ. Figures 1 shows in situ bright field TEM images of the growth of $\mathrm{Cu}_{2} \mathrm{O}$ islands as a function of time at $250^{\circ} \mathrm{C}$. As shown in Figures 1, one edge of the $\mathrm{Cu}_{2} \mathrm{O}$ triangular islands is along $<110>$ direction. The other two edges are along the directions with high indices which can vary from island to island. The light contrast feature around each $\mathrm{Cu}_{2} \mathrm{O}$ island is the result of lattice mismatch induced strain between $\mathrm{Cu}_{2} \mathrm{O}$ islands and $\mathrm{Cu}$ substrate. The atomic force microscopy (AFM) image of a typical $\mathrm{Cu}_{2} \mathrm{O}$ island formed at $200^{\circ} \mathrm{C}$ is shown in Figure 2 . The surface topology reveals that the oxide island has a rounded top and the island height is approximately equal to $2.6 \mathrm{~nm}$. According to the heteroepitaxial model of surface diffusion of oxygen, which was originally developed by Yang et al.[1], the saturation island density $N_{s}$ could follow an Arrhenius relationship with temperature and the 3-D island growth adopts a linear growth rate law, Figure 3 shows the saturation density of nuclei versus inverse oxidation temperature, where the activation energy, $E_{a}$, was determined to be $0.5 \pm 0.13 \mathrm{eV}$. Figure 4 shows the average oxidation island cross-section area and the island growth rates data at different temperatures and fit to the 3-D surface diffusion model. The experimental data indicates our initial analysis on the nucleation and growth of these three-dimensional $\mathrm{Cu}_{2} \mathrm{O}$ islands agree well with the heteroepitaxial model of surface diffusion of oxygen[1].

Our previous result of $\mathrm{Cu} 50 \% \mathrm{Au}$ in-situ oxidation has showed that double pyramid $\mathrm{Cu}_{2} \mathrm{O}$ islands form with cube-on-cube crystallographic orientation with respect to the film at temperature range from $550^{\circ} \mathrm{C}$ to $750^{\circ} \mathrm{C}$. Comparing with $\mathrm{Cu}(100)$ nano-oxidation, $\mathrm{Cu} 50 \% \mathrm{Au}$ alloy has much slower kinetics toward oxidation and longer incubation time at the same temperature[3]. The initial stage of oxidation of $\mathrm{CuAu}(100)$ at different component ( $\mathrm{CuAu} 5 \%, \mathrm{CuAu} 15 \%$ and $\mathrm{CuAu} 38 \%)$ at the higher temperature range $\left(800{ }^{\circ} \mathrm{C}-1000{ }^{\circ} \mathrm{C}\right)$ will be studied in order to extend our earlier studies. Other alloy such as $\mathrm{CuTi}$ and $\mathrm{CuTa}$ which are viewed as a novel scheme to prevent the formation of $\mathrm{Cu}$ oxide in the integrated circuit will also be studied. In these alloys the additive element $\mathrm{Ti}$ and $\mathrm{Ta}$ have limited solid solubility in $\mathrm{Cu}$ and may diffuse to the free surface. This outward diffusion of $\mathrm{Ti}$ and $\mathrm{Ta}$ will strongly influence the oxidation of $\mathrm{Cu}$, which will be investigated by our in situ UHV-TEM[4].

\section{References}

[1] J. C. Yang, M. Yeadon, B. Kolasa and J. M. Gibson, Scripta Materialia, 38 (8) (1998)1237. 
[2] L. Sun, J.C. Yang, Journal of Materials Research. 20 (7) (2005)1910.

[3] L.Wang, J. C. Yang, Journal of Materials Research, 20(7)(2005)1900

[4] This research project is funded by the National Science Foundation(\#0523205). The experiments were performed at the Materials Research Laboratory, University of Illinois at Urbana-Champaign, which is supported by the US Department of Energy (\#DEFG02-96- ER45439).
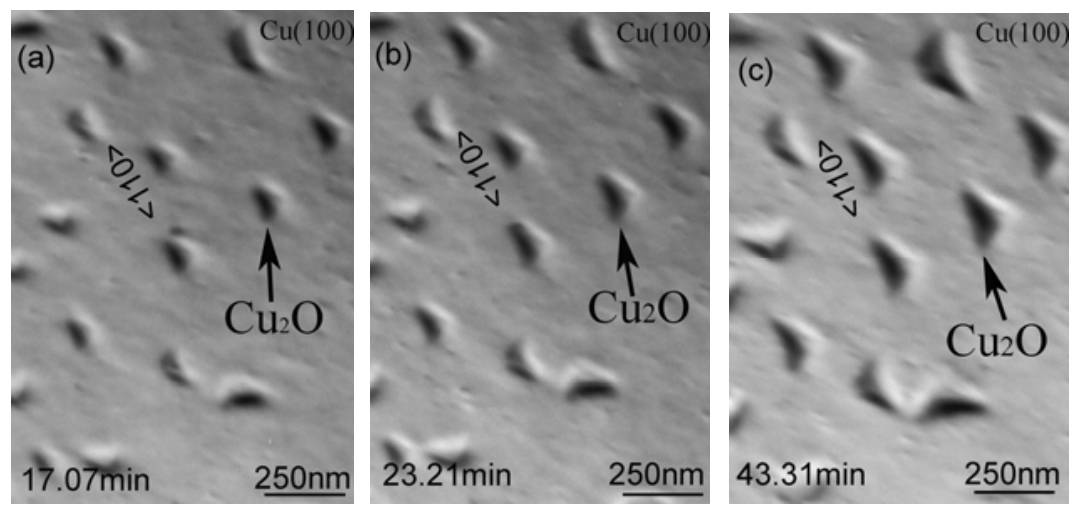

Figure 1 In situ bright field TEM images of $\mathrm{Cu}_{2} \mathrm{O}$ island growth on $\mathrm{Cu}(100)$ taken as a function of oxidation time, (a) $17.07 \mathrm{~min}$,(b) $23.21 \mathrm{~min} \mathrm{(c)} 43.31 \mathrm{~min}$ at constant oxygen partial pressure of $5 \times 10^{-4}$ torr and temperature of $250^{\circ} \mathrm{C}$.
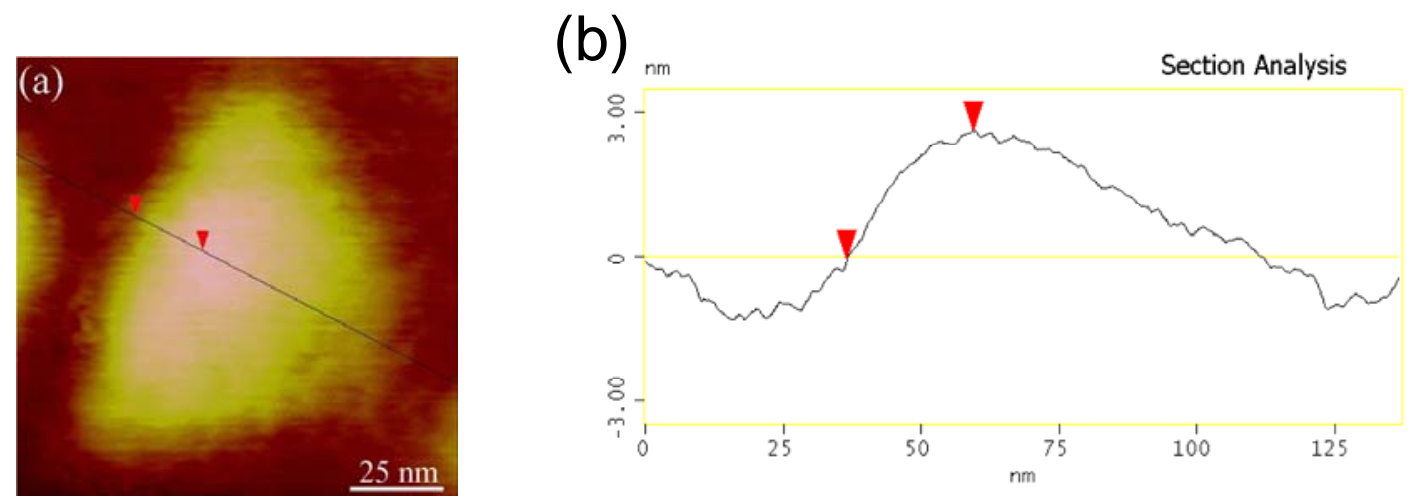

Figure 2 (a) AFM image of typical $\mathrm{Cu}_{2} \mathrm{O}$ islands formed on $\mathrm{Cu}(100)$ at $200^{\circ} \mathrm{C}$, and constant oxygen pressure of $5 \times 10^{-4}$ torr (b) cross-sectional profile drawn along the marked lines indicated in (a).

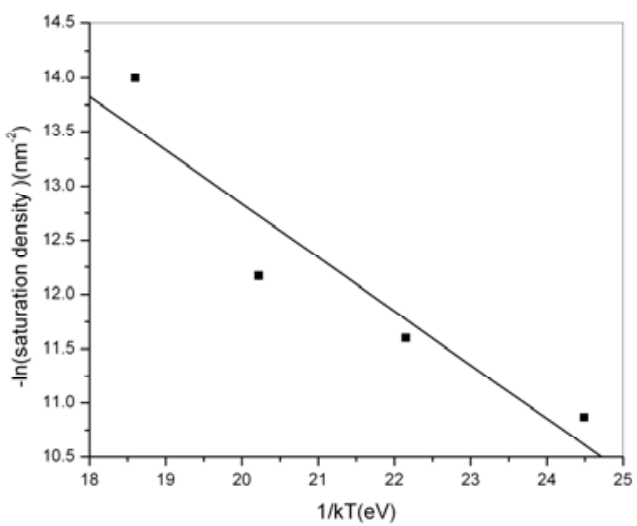

Figure $3 \mathrm{Cu}_{2} \mathrm{O}$ saturation island density versus inverse temperature. The absolute value of slope is the $E_{a}$ for the surface-limited process. $\mathrm{E}_{\mathrm{a}}=0.5 \mathrm{eV} \pm 0.13 \mathrm{eV}$

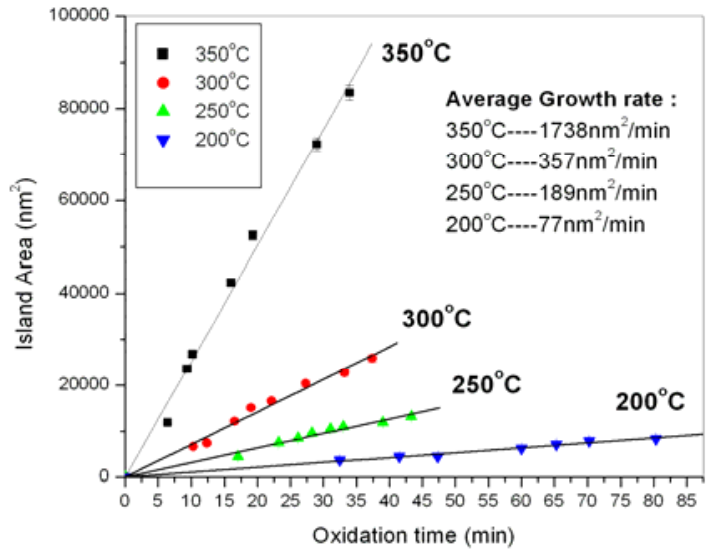

Figure 4 Comparison of the experimental data at the temperature $\boldsymbol{\bullet} 3500^{\circ} \mathrm{C}, \bullet 300^{\circ} \mathrm{C}, \boldsymbol{\Delta} 250^{\circ} \mathrm{C}, \boldsymbol{\nabla} 200^{\circ} \mathrm{C}$ and the theoretical function for the surface diffusion for the 3 - $\mathrm{D}$ growth of $\mathrm{Cu}_{2} \mathrm{O}$ islands. 